\title{
Reflexões sobre a relação entre resistência à insulina, diabetes mellitus e obesidade na adolescência à luz da literatura
}

\author{
Reflections on the relationship between insulin resistance, diabetes mellitus and obesity in \\ adolescents based on literature
}
Reflexiones sobre la relación entre la resistencia a la insulina, la diabetes mellitus y la obesidad en los adolescentes basado en la literatura

Vanessa Passos Oliveira ${ }^{1 *}$, Layonne de Sousa Carvalho ${ }^{1}$, Suzana Maria Rebêlo Sampaio Da Paz ${ }^{1}$, Marize Melo dos Santos ${ }^{1}$.

\section{RESUMO}

Objetivo: Revisar a literatura sobre os estudos que retratam a resistência à insulina, diabetes mellitus e obesidade na adolescência. Revisão bibliográfica: A insulina é um hormônio anabólico secretado pelas células-beta do pâncreas, tendo funções metabólicas que incluem: captação de glicose, aumento da síntese de proteínas, ácidos graxos e glicogênio. Alterações na função da insulina podem estar relacionadas a diversos fatores extrínsecos como mudanças nos hábitos alimentares e sedentarismo, e intrínsecos como, obesidade. O acúmulo excessivo de gordura corporal, principalmente a visceral, favorece a elevação de ácidos graxos livres na corrente sanguínea, os quais podem prejudicar a sinalização da insulina, diminuir a sensibilidade dos receptores nas membranas celulares e criar o quadro de resistência insulínica, que poderá levar ao surgimento de diabetes mellitus. Existe concordância no meio científico que a obesidade, especialmente em crianças e adolescentes, pode favorecer a identificação precoce de fatores que influenciam o surgimento de síndrome metabólica e diabetes mellitus. Considerações finais: A obesidade, principalmente a visceral, e o próprio DM estão relacionados ao maior risco de desenvolvimento de RI e outras complicações metabólicas em adolescentes.

Palavras-chave: Resistência à insulina, Diabetes mellitus, Obesidade, Adolescente.

\section{ABSTRACT}

Objective: To review the literature on studies that portray insulin resistance, diabetes mellitus and obesity in adolescence. Literature review: Insulin is an anabolic hormone secreted by pancreatic beta cells and has metabolic functions that include: glucose uptake, increased protein synthesis, fatty acids and glycogen. Changes in insulin function may be related to several extrinsic factors such as changes in eating habits and physical inactivity, and intrinsic factors such as obesity. Excessive accumulation of body fat, especially visceral fat, favors the elevation of free fatty acids in the bloodstream, which may impair insulin signaling, decrease receptor sensitivity in cell membranes and create insulin resistance, which may lead to increased insulin resistance. the onset of diabetes mellitus. There is agreement in the scientific world that obesity, especially in children and adolescents, may favor the early identification of factors that influence the onset of metabolic syndrome and diabetes mellitus. Final considerations: Obesity, especially visceral obesity, and DM itself are related to the higher risk of developing IR and other metabolic complications in adolescents.

Key words: Insulin resistance, Diabetes mellitus, Obesity, Adolescent.

\section{RESUMEN}

Objetivo: Revisar la literatura sobre estudios que muestran resistencia a la insulina, diabetes mellitus y obesidad en la adolescencia. Revisión bibliográfica: la insulina es una hormona anabólica secretada por las células beta pancreáticas y tiene funciones metabólicas que incluyen: absorción de glucosa, aumento de la

1 Universidade Federal do Piauí (UFPI),Teresina - PI. *E-mail: van_passos_oliveira@hotmail.com 
síntesis de proteínas, ácidos grasos y glucógeno. Los cambios en la función de la insulina pueden estar relacionados con varios factores extrínsecos, como los cambios en los hábitos alimenticios y la inactividad física, y factores intrínsecos como la obesidad. La acumulación excesiva de grasa corporal, especialmente la grasa visceral, favorece la elevación de ácidos grasos libres en el torrente sanguíneo, lo que puede afectar la señalización de la insulina, disminuir la sensibilidad del receptor en las membranas celulares y crear resistencia a la insulina, lo que puede conducir a una mayor resistencia a la insulina. La aparición de diabetes mellitus. Existe un acuerdo en el mundo científico de que la obesidad, especialmente en niños y adolescentes, puede favorecer la identificación temprana de factores que influyen en la aparición del síndrome metabólico y la diabetes mellitus. Consideraciones finales: la obesidad, especialmente la obesidad visceral, y la DM en sí están relacionadas con el mayor riesgo de desarrollar IR y otras complicaciones metabólicas en los adolescentes.

Palabras clave: Resistencia a la Insulina, Diabetes Mellitus, Obesidad, Adolescente.

\section{INTRODUÇÃO}

A adolescência é um período de transição da infância para a fase adulta em que ocorrem importantes mudanças biológicas, cognitivas, emocionais e sociais. As modificações nos níveis de estrogênio e testosterona durante a puberdade são responsáveis por alterações na composição corporal e no perfil bioquímico dos adolescentes, com diferenças significativas entre os sexos, uma vez que os meninos apresentam maior proporção de massa livre de gordura e as meninas, uma maior quantidade de massa de gordura (WORLD HEALTH ORGANIZATION, 2005).

A puberdade é um período transitório e fisiológico de resistência à insulina (RI), parcialmente explicado pelo aumento dos hormônios esteroides sexuais e do hormônio de crescimento, responsáveis pelo desenvolvimento das características sexuais secundárias, aumento acentuado da estatura e mudança da composição corporal (HANNON TS, et al., 2006).

Nessa fase da vida também se desenvolvem atitudes e práticas comportamentais relacionadas à saúde associadas ao desenvolvimento da maioria das doenças crônicas não transmissíveis e que apresentam grande chance de permanecer ao longo da vida, podendo influenciar o estado de saúde na idade adulta (OLIVEIRA-CAMPOS M, et al., 2014; FRANKLIN TAF, et al., 2018).

Apesar do diabetes mellitus (DM) acometer principalmente indivíduos a partir da quarta década de vida, tem sido descrito que, em alguns países, há o aumento na sua incidência em crianças e jovens (RAO PV, 2015). Estudo realizado em estados americanos entre 2002 e 2012, retratou aumento de 7\% ao ano na prevalência de DM nesta população (MAYER-DAVIS EJ, et al., 2017).

O diabetes mellitus vem crescendo em decorrência de vários fatores, como o aumento na taxa de urbanização e da expectativa de vida, a industrialização, maior consumo de dietas hipercalóricas e ricas em hidratos de carbono de absorção rápida, sedentarismo, sendo também necessário considerar a maior sobrevida da pessoa diabética (ORTIZZ MCA e ZANETTI ML, 2001). Dentre os adolescentes, os principais fatores associados ao DM são os genéticos e o estilo de vida característico da adolescência, como alimentação rica em gorduras e açúcares refinados, além do sedentarismo (ROMUALDO MC, et al., 2014; GOBATO AO, et al., 2014; SBD, 2016).

Assim como em adultos, o diabetes mellitus em jovens é diagnosticado quando há resistência hepática à insulina e alteração na função das células $\beta$, com aumento da produção hepática de glicose, da filtração renal e da lipólise (DEFRONZO RA, TRIPATHY D, 2009). Da mesma forma, os fatores de risco para DM são iguais entre a população jovem e adulta, são eles: história familiar da doença, obesidade, sedentarismo, diagnóstico prévio de pré-diabetes ou diabetes mellitus gestacional (DMG) e presença de componentes da síndrome metabólica, tais como hipertensão arterial, dislipidemia, além da resistência à insulina (SBD, 2017).

Nessa perspectiva, diante da complexidade e pouco aprofundamento na literatura científica sobre a temática, este artigo teve por objetivo refletir sobre estudos que retratam a relação da resistência à insulina, diabetes mellitus e obesidade na adolescência. 


\section{REVISÃO BIBLIOGRÁFICA}

\section{Mecanismos de ação da insulina na obesidade}

A insulina é um hormônio anabólico secretado pelas células $\beta$ do pâncreas; sua síntese é estimulada pelo aumento da glicose sanguínea após refeições, tendo funções metabólicas que incluem: captação de glicose, aumento da síntese de proteínas, ácidos graxos e glicogênio, levando a uma redução na produção hepática de glicose e inibição da lipólise, atuando no músculo esquelético, fígado e tecido adiposo (LIN HV, et al., 2011). O receptor da insulina é uma proteína hetorotetramérica, contento duas subunidades $\alpha$ extracelulares dispostas simetricamente e que se ligam, cada uma delas, por pontes dissulfeto a uma unidade $\beta$ transmembrana, com domínio intracelular, com atividade quinase intrínseca (GONZALEZ E, MCGRAW TE, 2006).

A sinalização da insulina ocorre quando esta se liga ao seu receptor na região extracelular da subunidade $\alpha$, ocasionando a ativação da tirosina quinase da subunidade $\beta$ e ativando, através de fosforilação, diversas proteínas substratos intracelulares do receptor de insulina, ativando cascatas de sinalização como fosfatidilinositol 3-quinase (PI3q), que modulam a ação da insulina. A sinalização da insulina favorece o movimento e fixação das vesículas contendo a proteína transportadora de glicose GLUT-4 na membrana plasmática, proporcionando a captação da glicose (ABBAS AK, et al., 2010).

Alterações na função da insulina podem estar relacionadas a diversos fatores extrínsecos como mudanças nos hábitos alimentares e sedentarismo, e intrínsecos como, obesidade (PAULINO MT, MALDONADO RR, 2014). A resistência à insulina é uma das mais importantes repercussões encontradas em obesos, indicando ser o fator que propicia o desencadeamento das demais alterações metabólicas (ROMUALDO MC, et al., 2014).

Na obesidade, a capacidade da insulina de estimular a utilização da glicose fica comprometida, uma vez que as citocinas pró-inflamatórias produzidas pelo tecido adiposo são capazes de reduzir a fosforilação dos substratos do receptor de insulina (IRS) e a sinalização da PI3K, com redução da síntese e translocação do GLUT-4 para a membrana celular (CHISSINI RBC, et.al., 2015). Consequentemente a estes mecanismos, a supressão da lipólise é prejudicada, elevando a circulação de ácidos graxos livres que, ao alcançarem os tecidos sensíveis à insulina, inibem sua ação ligando-se aos receptores e modificando a sinalização celular, alterando ainda mais o transporte de glicose para os tecidos-alvo (HOLLAND WL, et al., 2011; ROMUALDO MC, et al., 2014).

Níveis elevados de ácidos graxos livres prejudicam a sinalização de insulina por ativarem proteínas de membrana plasmática denominados TLR-4 (toll like receptors 4), desencadeando a ativação de vias inflamatórias que vão interferir na captação de glicose (DASU MR, JIALAL I, 2010). Induzem, assim, um recrutamento de macrófagos aos adipócitos e liberação de citocinas pró-inflamatórias como fator de necrose tumoral $\alpha($ TNF- $\alpha$ ) e a interleucina- 6 (IL-6), estando relacionada também a outras consequências metabólicas, como disfunção endotelial, hipertrofia vascular, rigidez arterial e elevação da pressão sanguínea (SOLTANI Z, et al., 2015; VÁZQUEZ-JIMÉNEZ JG, et al., 2017).

Diante dessas vias de sinalização, ressalta-se que indivíduos obesos possuem maior risco de desenvolvimento de resistência à insulina e outras complicações metabólicas importantes, como o diabetes mellitus tipo 2.

\section{Obesidade, diabetes mellitus e resistência à insulina em adolescentes}

O diabetes mellitus consiste em um distúrbio metabólico com forte herança familiar, caracterizado por hiperglicemia persistente decorrente de deficiência na produção de insulina ou na sua ação, ou em ambos os mecanismos, ocasionando complicações a longo prazo (SBD, 2017). É importante ressaltar que a resistência à ação da insulina não é sinônimo de pré-diabetes, uma vez que para desenvolver esta doença, além do quadro de RI instalado, necessita-se de deficiência na secreção pancreática de insulina. Porém, a Associação Americana de Diabetes aponta que 1/3 dos pacientes com RI desenvolvem DM. Indivíduos com história familiar de primeiro grau e/ou obesidade são expostos ao maior risco (ADA, 2015). 
É sabido que a RI pode ser fisiológica (gravidez, puberdade, envelhecimento) ou patológica (SBD, 2017). Essa última envolve a interação de múltiplos fatores, incluindo genética, sexo, idade, sedentarismo, hábitos alimentares inadequados, tabagismo, etilismo, excesso de peso e/ou de gordura corporal e presença de alterações como dislipidemias e hipertensão arterial (DESPRES JP, 2012).

Ressalta-se a obesidade como importante fator de risco para desenvolvimento de RI e DM em crianças e adolescentes, e a presença de alterações metabólicas ainda na infância, o que é preocupante, já que a resistência à insulina e as complicações relacionadas podem ser agravadas durante a adolescência, devido à diminuição fisiológica da sensibilidade à insulina, que está associada ao desenvolvimento puberal (FARIA ER, et al., 2014; SILVA AOB, et al., 2018).

Pesquisas têm demonstrado a relação entre variáveis associadas ao excesso de peso, como o índice de massa corporal (IMC), circunferência da cintura (CC) e circunferência do pescoço (CP), com a presença de resistência à insulina, diabetes mellitus e síndrome metabólica no grupo dos adolescentes (ROMUALDO MC, et al., 2014; GOBATO AO, et al., 2014; RODRIGUES CN, et al., 2015; LEAL JDV, et al., 2016).

Durante a puberdade, essa relação pode estar ligada a um mecanismo que aumenta o efeito anabólico da insulina e do hormônio do crescimento durante o rápido crescimento somático que ocorre neste período, levando a alterações na distribuição de gordura (KURTOGLU S, et al., 2010; SINGH Y, et al., 2013). A Associação Americana de Diabetes (ADA) estabeleceu critérios para classificar crianças e adolescentes com risco de desenvolver DM2, sendo eles: sobrepeso/obesidade com IMC igual ou acima do percentil 85 e a presença de dois ou mais fatores para DM 2 (histórico familiar de DM, alto risco de raça/ etnia, resistência insulínica, hipertensão arterial e a presença da acantose nigricans (ADA, 2000).

Meta-análise realizada por Hamiel OP e Zeitler P (2005) demonstrou aumento da incidência global do DM2 em crianças e adolescentes e indicou associação entre esses achados e a elevação nos casos de obesidade em jovens e crianças nos EUA, Ásia, Nova Zelândia, Austrália, parte da Europa, América do Sul e Canadá. Pesquisadores observaram que adolescentes de Viçosa-MG, que tinham excesso de peso, apresentavam 4,5 vezes a maior a chance de ter o índice que avalia a resistência à insulina, HOMA-IR (Homeostasis Model Assessment-Insulin Resistance), alterado, e que os seus valores eram maiores nas adolescentes com maior percentual de gordura (SERRANO HM, et al., 2010).

Em amostra de 80 adolescentes obesos pós-púberes, divididos em obesos com menor e maior grau de obesidade, encontrou-se que aqueles com maior grau de obesidade apresentavam maiores frequências de alterações na glicemia, HOMA-IR e pressão arterial, demonstrando que o grau de obesidade pode ser determinante no aparecimento de alterações clínicas e metabólicas (LAVRADOR MSF, et al., 2011). Além disso, a resistência à insulina esteve presente em $41 \%$ dos adolescentes obesos atendidos pelo Sistema Único de Saúde, em Campina Grande-PB (ROMUALDO MC, et al., 2014), e em 29,1\% dos adolescentes atendidos no Ambulatório de Obesidade na Criança e no Adolescente, do Hospital de Clínicas da Faculdade de Ciências Médicas, da Universidade Estadual de Campinas (GOBATO AO, et al., 2014).

No Brasil, foram encontradas prevalências de $49,2 \%, 58,3 \%$ e $70,6 \%$ de diabetes mellitus entre adultos do sexo feminino, atribuíveis ao sobrepeso, à obesidade e ao excesso de peso, respectivamente. Entre os homens, esses percentuais foram 40,5\%, 45,4\% e 60,3\%, respectivamente (FLOR LS, et al., 2015). Assim, existe concordância no meio científico que a obesidade, especialmente em crianças e adolescentes, pode favorecer a identificação precoce de fatores que influenciam o surgimento de síndrome metabólica, resistência insulínica e diabetes mellitus (ROMUALDO MC, et al., 2014; FLOR LS, et al., 2015; CORRÊA RS, et al., 2017). A eclosão de casos de diabetes tipo 2 na infância e na adolescência está fortemente associado à transição nutricional que vem ocorrendo nas últimas décadas, com aumento no consumo de ultraprocessados, maior acesso à alimentação tipo fast-food e, paralelamente a isso, redução do consumo de alimentos in natura, como frutas e legumes que levou a uma epidemia mundial de obesidade (VASCONCELOS HCA, 2010; SBD, 2017).

Diante dos estudos supracitados que sinalizam os adolescentes como um grupo vulnerável às alterações do metabolismo da glicose, é imprescindível a articulação de políticas públicas voltadas para este público. 
Devem ser priorizadas abordagens focadas na mudança do estilo de vida e adoção de práticas alimentares saudáveis, uma vez que constituem fatores modificáveis e que podem repercutir de maneira positiva na melhora da qualidade de vida, reduzindo os riscos de morbimortalidade os quais estes indivíduos estão expostos.

\section{CONSIDERAÇÕES FINAIS}

A obesidade está relacionada ao maior risco de desenvolvimento de resistência insulínica, diabetes mellitus e outras complicações metabólicas em adolescentes. O crescimento dos casos obesidade na adolescência decorre, principalmente do grande consumo de produtos industrializados em dietas hipercalóricas, além do sedentarismo característico da vida moderna. Logo, o tratamento de primeira escolha para o diabetes mellitus tipo 2 na adolescência se inicia com a prática de atividades físicas e com a alteração nos hábitos alimentares para dietas saudáveis, contribuindo na redução dos fatores de risco. Deste modo, recomenda-se que ações voltadas para a prevenção de obesidade sejam direcionadas para crianças e adolescentes, visto que mudanças no comportamento alimentar nesta fase diminuem o risco de doenças e agravos relacionados ao acúmulo de gordura corporal na fase adulta.

\section{REFERÊNCIAS}

1. ABBAS AK, et al. Patologia - Bases Patológicas das Doenças. Tradução de Fernandes, Patrícia Dias et al. 8. ed. Rio de Janeiro: Elsevier, 2010. 1480p.

2. AMERICAN DIABETES ASSOCIATION (ADA). Position statement. Diabetes Care. 2015; 38 (1): S20-S30.

3. AMERICAN DIABETES ASSOCIATION (ADA). Type 2 diabetes in children and adolescents. Pediatrics. 2000;105(3):671-680.

4. CHISSINI RBC, et al. Obesidade na infância e adolescência: associação da inflamação e resistência à insulina com alterações metabólicas. Brazilian Journal of Health and Biomedical Sciences, 2015; 14(3): 41-49.

5. CORRÊA RS, et al. Padrões alimentares de escolares: existem diferenças entre crianças e adolescentes? Ciência \& Saúde Coletiva, 2017; 22(2):553-562.

6. DASU MR, JIALAL I. Free fatty acids in the presence of high glucose amplify monocyte inflammation via toll-like receptors. American Journal of Physiology-Endocrinology and Metabolism, 2010; 300:145-154.

7. DEFRONZO RA, TRIPATHY D. Skeletal muscle insulin resistance is the primary defect in type 2 diabetes. Diabetes care, 2009; 32(2): S157-S163.

8. DESPRES JP. Obesidade abdominal e doença cardiovascular: a inflamação é o elo perdido? Jornal of Cardiology, 2012; 28(6): 642-652.

9. FARIA ER, et al. Resistência à insulina e componentes da síndrome metabólica, análise por sexo e por fase da adolescência. Arquivos Brasileiros de Endocrinologia e Metabolismo, 2014; 58(6): 610-618.

10. FLOR LS, et al. Carga de diabetes no Brasil: fração atribuível ao sobrepeso, obesidade e excesso de peso. Revista de Saúde Pública, 2015; 49(1): 1-11.

11. FRANKLIN TAF, et al. Comportamentos de Risco a Saúde em Adolescentes Residentes em Município de Pequeno Porte. Revista Cuidado é Fundamental, 2018; 10(3): 704-710.

12. GOBATO AO, et al. Síndrome metabólica e resistência à insulina em adolescentes obesos. Revista Paulista de Pediatria, 2014; 32(1): 55-62.

13. GONZALEZ E, MCGRAW TE. Insulin signaling diverges into akt-dependent and independente signals to regulate the recruitment docking and the fusion of GLUT4 vesicles to the plasma membrane. Mollecular Biology of the Cell, 2006;17:4484-4493.

14. HAMIEL OP, ZEITLER P. The global spread of type 2 diabetes mellitus in children and adolescents. The Journal of Pediatrics. 2005; 146(5):693-700.

15. HANNON TS, et al. Longitudinal study of physiologic insulin resistance and metabolic changes of puberty. Pediatric Research, 2006; 60(6):759-763.

16. HOLLAND WL, et. al. Lipid-induced insulin resistance mediated by the proinflammatory receptor TLR4 requires saturated fatty acid-induced ceramide biosynthesis in mice. The Journal of clinical investigation, 2011; 121(5): 18581870.

17. KURTOGLU S, et al. Insulin Resistance in Obese Children and Adolescents: HOMA- IR Cut- Off Levels in the Prepubertal and Pubertal Periods. Journal of clinical Research in Pediatric Endocrinology, 2010; 2(3): $100-106$. 
18. LAVRADOR MSF, et al. Riscos cardiovasculares em adolescentes com diferentes graus de obesidade. Arquivos Brasileiros de Cardiologia, 2011;96(3): 205-211.

19. LEAL JDV, et al. Perfil clínico e metabólico e sua relação com resistência à insulina entre escolares. Revista da Rede de Enfermagem do Nordeste, 2016;17(3):393-400.

20. LIN HV, et al. Diabetes in mice with selective impairment of insulin action in Glut4-expressing tissues. Diabetes, 2011;60(3):700-709.

21. MAYER-DAVIS EJ, et al. Incidence trends of type 1 and type 2 diabetes among youths, 2002-2012. The new England Journal of Medicine. 2017; 376(15): 1419-1429.

22. OLIVEIRA-CAMPOS M, et al. Comportamento sexual em adolescentes brasileiros, Pesquisa Nacional de Saúde do Escolar (PeNSE 2012). Revista Brasileira de Epidemiologia. 2014;17(1): 116-130.

23. ORTIZ MCA, ZANETTI ML. Levantamento dos Fatores de Risco para Diabetes Mellitus tipo 2 em uma Instituição de Ensino Superior. Revista Latino-americana Enfermagem. 2001; 9(3):58-63.

24. PAULINO MT, MALDONADO RR. Diabetes mellitus e resistência à insulina. Interciência e Sociedade, 2014; 3(2): 2735.

25. RAO PV. Type 2 diabetes in children: clinical aspects and risk factors. Indian Journal of Endocrinology and Metabolism, 2015;19(1): 47-50.

26. RODRIGUES CN, et al. Resistência à insulina e diabetes tipo 2: uma análise transversal em um programa de intervenção nutricional. Revista de Atenção à Saúde. 2015;13(46): 11-22.

27. ROMUALDO MC, et al. Resistência à insulina em crianças e adolescentes obesos. Jornal de Pediatria, 2014; 90(6): 600-607.

28. SERRANO HM, et al. Composição corpórea, alterações bioquímicas e clínicas de adolescentes com excesso de adiposidade. Arquivo Brasileiro de Cardiologia. 2010;95(1): 464-472.

29. SILVA AOB, et al. Relação da alimentação com surgimento precoce da obesidade e diabetes mellitus tipo 2 em crianças e adolescentes. Revista Eletrônica Acervo Saúde. 2018; 18(1): 3-6.

30. SINGH Y, et al. A study of insulin resistance by HOMA-IR and its cut-off value to identify metabolic syndrome in urban Indian adolescents. Journal of Clinical Research İn Pediatric Endocrinology. 2013; 5(4): 245-251.

31. SOCIEDADE BRASILEIRA DE DIABETES (SBD). Diretrizes da Sociedade Brasileira de Diabetes (2015-2016). Análise dos Marcadores de Resistência à Insulina na Clínica Diária. São Paulo: A.C. Farmacêutica, 2016.

1. 32.SOCIEDADE BRASILEIRA DE DIABETES (SBD). Diretrizes da Sociedade Brasileira de Diabetes 2017-2018/ Organização José Egídio Paulo de Oliveira, Renan Magalhães Montenegro Junior, Sérgio Vencio. São Paulo: Editora Clannad, 2017.

32. SOLTANI Z, et al. The impacts of obesity on the cardiovascular and renal systems: cascade of events and therapeutic approaches. Current hypertension reports. 2015;17(2):1-14.

33. VASCONCELOS HCA, et al. Fatores de risco para diabetes mellitus tipo 2 entre adolescentes. Revista da Escola de Enfermagem da USP. 2010;4(4):881-7.

34. VÁZQUEZ-JIMÉNEZ JG, et al. El papel de los ácidos grasos libres en la resistencia a la insulina. Gaceta medica de Mexico. 2017;153(7): 852-863.

35. WORLD HEALTH ORGANIZATION. Nutrition in adolescence - Issues and challenges for the health sector: issues in adolescent health and development. 2005; 115p. 